\title{
Clues for the development of food-based dietary guidelines: how are dietary targets being achieved by UK consumers?
}

\author{
Steven J. Wearne* and Michael J. L. Day \\ Joint Food Safety and Standards Group, Ministry of Agriculture, Fisheries and Food, Ergon House, clo Nobel House, \\ 17 Smith Square, London SWIP 3JR, United Kingdom
}

\begin{abstract}
Expert scientific advice to the UK Government has been translated into eight general dietary guidelines, which form the core of population-based dietary advice in the UK and are supplemented by a food selection guide showing the types and proportions of foods needed for a balanced and healthy diet. Data from the Dietary and Nutritional Survey of British Adults were used to identify statistically significant differences between subgroups of the study population that met, or failed to meet, population nutritional goals for intakes of total fat, saturated fat and dietary fibre. Several eating habits - including greater consumption of starchy foods (particularly wholemeal varieties), greater consumption of fruit and the substitution of reduced-fat milk for whole-fat milk - were shared by the subgroups that met each of the nutritional goals. This analysis provides clues for any future refinement of food-based dietary guidelines.
\end{abstract}

Dietary fat: Dietary fibre: Dietary patterns: Food-based dietary guidelines: Saturated fat

Dietary guidelines, however they are presented and communicated, need to be based on a robust assessment of the available scientific information on diet and the maintenance of good health. This assessment needs to include an evaluation of the prevailing diet and nutrient intake compared to any recommended optimum. Any dietary guidelines developed as a result need then to be pragmatic - a stepwise approach may be needed to address any large disparities between actual and ideal dietary habits.

In the UK, scientific assessments are undertaken by the Committee on Medical Aspects of Food and Nutrition Policy (COMA), a committee of independent experts, which advises the Department of Health. COMA completed an extensive appraisal of the relevant science in 1991 with the publication of its report recommending Dietary Reference Values (DRVs) for a wide range of macro- and micronutrients (Department of Health, 1991). For fruits and vegetables, the World Health Organization recommendation of $400 \mathrm{~g} / \mathrm{d}$ is roughly equivalent to the consumption of five portions per day, which is advised as a minimum target for UK consumers (World Health Organization, 1990; Health Education Authority, 1997).

The DRVs for total fat and saturated fat were formalized into nutritional goals by the 1992 'Health of the Nation' policy paper (Department of Health, 1992) and formed part of overall strategies to reduce the incidence of cardiovascular disease. In each case, the target was for population averages to match the DRVs by 2005: 'to reduce the average percentage of food energy derived by the population from saturated fatty acids by at least $35 \%$ by 2005 (from $17 \%$ in 1990 to no more than $11 \%$ )'; and 'to reduce the average percentage of food energy derived by the population from total fat by at least $12 \%$ by 2005 (from about $40 \%$ in 1990 to no more than $35 \%$ )'. Diet continues to be a part of the focus for action in meeting the new targets for reductions in cancer and cardiovascular disease proposed in the 1998 consultation paper 'Our Healthier Nation' (Department of Health, 1998).

Advice from COMA, including that on DRVs, has been translated into the following eight guidelines, which are disseminated through a guide for nutrition educators (Health Education Authority, 1997) and a series of posters and booklets aimed at the general public: (i) enjoy your food; (ii) eat a variety of different foods; (iii) eat the right amount to be a healthy weight; (iv) eat plenty of foods rich in starch and fibre; (v) eat plenty of fruit and vegetables; (vi) don't eat too many foods that contain a lot of fat; (vii) don't have sugary foods and drinks too often; and (viii) if you drink alcohol, drink sensibly. In order to make healthy eating easier to understand, the guideline 'eat a variety of different foods' is supplemented by a food selection guide, entitled The Balance of Good Health, which is in the form of a tilted plate and shows the types and proportions of foods needed 
for a balanced and healthy diet. The following five categories of foods are represented: fruit and vegetables (33\% of the area of the plate); bread, other cereals and potatoes (33\%); milk and dairy foods (15\%); meat, fish and alternatives $(12 \%)$; and foods containing fat, foods containing sugar $(8 \%)$. Research has shown that exposure to such a food selection guide, even if only briefly, can have a significant effect on people's understanding of healthy eating messages - particularly on how foods can be divided into groups, and the proportion of foods from the different groups that should be consumed (Hunt et al. 1995). The food selection guide was introduced in July 1994 and, although not the only model of its kind (World Health Organization, 1998), it has become widely accepted and used, mainly as a result of an active ongoing promotion to health professionals and educators by the Health Education Authority (Research and Auditing Services Ltd, 1997).

The UK national healthy eating guidelines are similar to those proposed for many other Northern European countries. However, there are also some marked differences between national guidelines. This is unsurprising, as the variations in dietary habits across Europe are such that it is more appropriate and effective to address these issues at a national or even sub-national level (World Health Organization, 1998). For example, in the UK the emphasis put on the need to reduce consumption of fat and saturated fat, and to increase consumption of fruit and vegetables, would be inappropriate in Mediterranean countries where consumption of these nutrients and foods by large subgroups of the population is much closer to what is considered to be ideal. Therefore, the materials produced in the UK and the way in which the message is disseminated reflect not only the particular nutritional needs and status of various population groups, on which there is a large degree of international consensus, but also the particular cultural and other circumstances of the UK.

Recently, proposals have been made for international collaboration in the setting of food-based dietary guidelines (World Health Organization, 1998). This paper gives an insight into the dietary habits adopted by UK consumers who are successful in meeting current nutritional benchmarks, thereby providing clues for the development of future food-based dietary guidelines that would be relevant to the UK.

\section{Experimental methods}

\section{Source of dietary intakes data}

Dietary intakes data used in this paper are taken from the Dietary and Nutritional Survey of British Adults aged 16 to 64 years (Gregory et al. 1990). Although this is now somewhat dated, the field work having been conducted in 1986-1987, the $7 \mathrm{~d}$ weighed diary record methodology used in the survey and the relatively large sample size of 2197 British adults provide robust data on dietary habits at that time. An assessment of the quality of the data from the survey in terms of recorded energy intakes concluded that these were broadly consistent with those from other surveys of the general population. Although there was evidence that the recorded intake under-represented habitual intakes, there was no evidence from the data that under-reporting or modification of habitual diets were confined to particular subgroups of the sample (Gregory et al. 1990).

\section{Sources of nutritional goals}

The goals for total fat and saturated fat used in this paper are those recommended by COMA (Department of Health, 1991). For dietary fibre, there is the complication of the DRV being expressed in terms of non-starch polysaccharide and the dietary survey data being based on fibre measured by the Southgate method (Southgate, 1969). For the purposes of this paper, we have assumed that the DRV is equivalent to $50 \mathrm{~g} / \mathrm{d}$ of Southgate fibre.

\section{Definition of food intake patterns}

The objective of this paper is the identification of dietary habits adopted by groups of UK consumers who are successful in meeting current recommended nutrient intakes. The first step in this process is the identification of groups in the study population who (i) met the relevant nutritional goal(s) over the study period (these are termed 'compliers'), and (ii) did not meet the nutritional goal(s) (these are termed 'non-compliers').

The approach taken has been described elsewhere (MAFF, 1994). In summary, the intakes of total fat or saturated fat by each of the individuals in the study group were ranked from lowest and highest. Starting from the lowest intake, successive individuals were added until the addition of one more subject caused the average of the group to exceed the DRV. The approach taken for dietary fibre was the same, except intakes were ranked from highest to lowest and successive individuals added until addition of one more subject caused the average intake to fall below the DRV. This approach gave, in each case, the maximum size of subgroup of 'compliers' who met the population target, although some of the individuals in the group of 'compliers' for fat or saturated fat would have intakes that exceeded the nutritional goal, and some of the individuals in the group of 'compliers' for dietary fibre would have intakes that fell short of this nutritional goal.

T-tests were used to compare the consumption of each food type by the subgroup of 'compliers' to consumption by the subgroup of 'non-compliers' and statistically significant differences $(P<0.05)$ were noted. These comparisons are indicative of where the most significant differences lie, but should be interpreted with some caution as they do not take into account the correlations that result from the inherent causative nature of, for example, increased highfibre breakfast cereal consumption on overall intakes of dietary fibre. This approach has two advantages over the use of set quantiles of the study population to address the objective of this paper: (i) the division of the study population into subgroups is not arbitrary, instead it explicitly uses the DRV to differentiate between the subgroups; and (ii) the approach is consistent with the theoretical derivation of DRVs for total fat, saturated fat and dietary fibre, which are population averages, not targets for individuals (Department of Health, 1991).

Table 1 characterizes the study population in terms of average intakes of total fat, saturated fat, fibre and consumption of fruit and vegetables (Gregory et al. 1990). It 
Table 1. Measures of intakes of total fat, saturated fat and fibre and of consumption of fruit and vegetables by the study population, and their comparison to Dietary Reference Values and nutritional goals for these nutrients and foods

\begin{tabular}{|c|c|c|c|c|}
\hline & Total fat & Saturated fat & Dietary fibre & Fruit and vegetables \\
\hline
\end{tabular}

ND: not determined.

also provides details of the number of individuals who met the DRVs, and the numbers in the 'compliers' and 'noncompliers' subgroups that were generated for each nutrient by application of the above methodology.

\section{Results}

\section{Total fat intakes}

There were statistically significant $(P<0 \cdot 05)$ differences in consumption of a number of food groups between 'compliers' and 'non-compliers' (Table 2). In general, the subgroup complying with the dietary target on total fat tended, unsurprisingly, to follow the general healthy eating advice disseminated by Government and health education agencies.

Both men and women 'compliers' consumed significantly more reduced-fat milk (in terms of both the proportion of consumers and their mean intake) and less whole milk than 'non-compliers'. 'Compliers' who consumed less of other animal products (meat, poultry and eggs), more fruit, fewer biscuits, cakes, pastries and savoury snacks, and more alcoholic drinks than 'non-compliers'. Women 'compliers' (but not men) ate significantly more starchy foods and sugary foods than 'non-compliers'. With the exception of milk and alcoholic drinks, these differences in absolute terms are relatively small; for example the mean consumption of meat, poultry and eggs by 'non-compliers' was just $15 \%$ higher than the mean consumption of these foods by 'compliers'.

\section{Saturated fat intakes}

Dietary patterns of 'compliers' and 'non-compliers' are compared in Table 3. Statistically significant differences in habits between the two subgroups, defined in terms of saturated fat intakes, were similar to those observed when the subgroups were defined in terms of total fat intakes.

Both men and women 'compliers' consumed significantly less whole milk, in terms of both the mean intake and proportion of consumers and their mean intake, than 'noncompliers'. Women 'compliers' (but not men) consumed significantly more reduced-fat milk than 'non-compliers'. As a result, the consumption of all dairy products was significantly higher in men 'non-compliers' than in men 'compliers'.
'Compliers' of both sexes ate significantly less meat and alternatives than 'non-compliers', with the greatest part of this difference being due to differences in consumption of meat and poultry between the groups. They also ate significantly fewer biscuits, cakes and pastries.

Women 'compliers' (but not men) ate significantly fewer savoury snacks and chips than 'non-compliers'. Men 'compliers' (but not women) consumed significantly more alcoholic drinks than 'non-compliers', with the greatest part of this difference due to differences in consumption of beverages in the 'beer, cider and perry' group.

\section{Dietary fibre intakes}

Table 4 compares the dietary habits of subgroups of men and women which as a whole met or did not meet the target of at least $30 \mathrm{~g} / \mathrm{d}$ of dietary fibre (measured by the Southgate method). Although the overall food groups used in this analysis are the same as those used for comparisons between groups based on total fat and saturated fat intakes, these food groups have been split into different subgroups that are more informative for this particular analysis (e.g. splitting breakfast cereals into high-fibre and other varieties). A number of statistically significant differences were again observed between 'compliers' and 'non-compliers'.

'Compliers' of both sexes ate significantly more starchy foods than 'non-compliers'. Within this group of foods, there were significantly higher levels of consumption by 'compliers', both in terms of the proportion of consumers and their mean intake, for wholemeal bread and high-fibre breakfast cereals. For women 'compliers', the analysis was consistent with these higher levels of consumption of wholemeal bread being caused by substitution for white bread. Significantly higher consumption of other breads and potatoes (other than fried) by men and women 'compliers', when compared to 'non-compliers', also contributed to the observed differences in levels of consumption of starchy foods between the two groups. The proportion of consumers and their mean intakes also contributed to significantly higher levels of consumption of fruit and of nuts and pulses by both men and women 'compliers'. Although there were no differences in the proportion of 'compliers' and 'noncompliers' who consumed vegetables during the $7 \mathrm{~d}$ study 


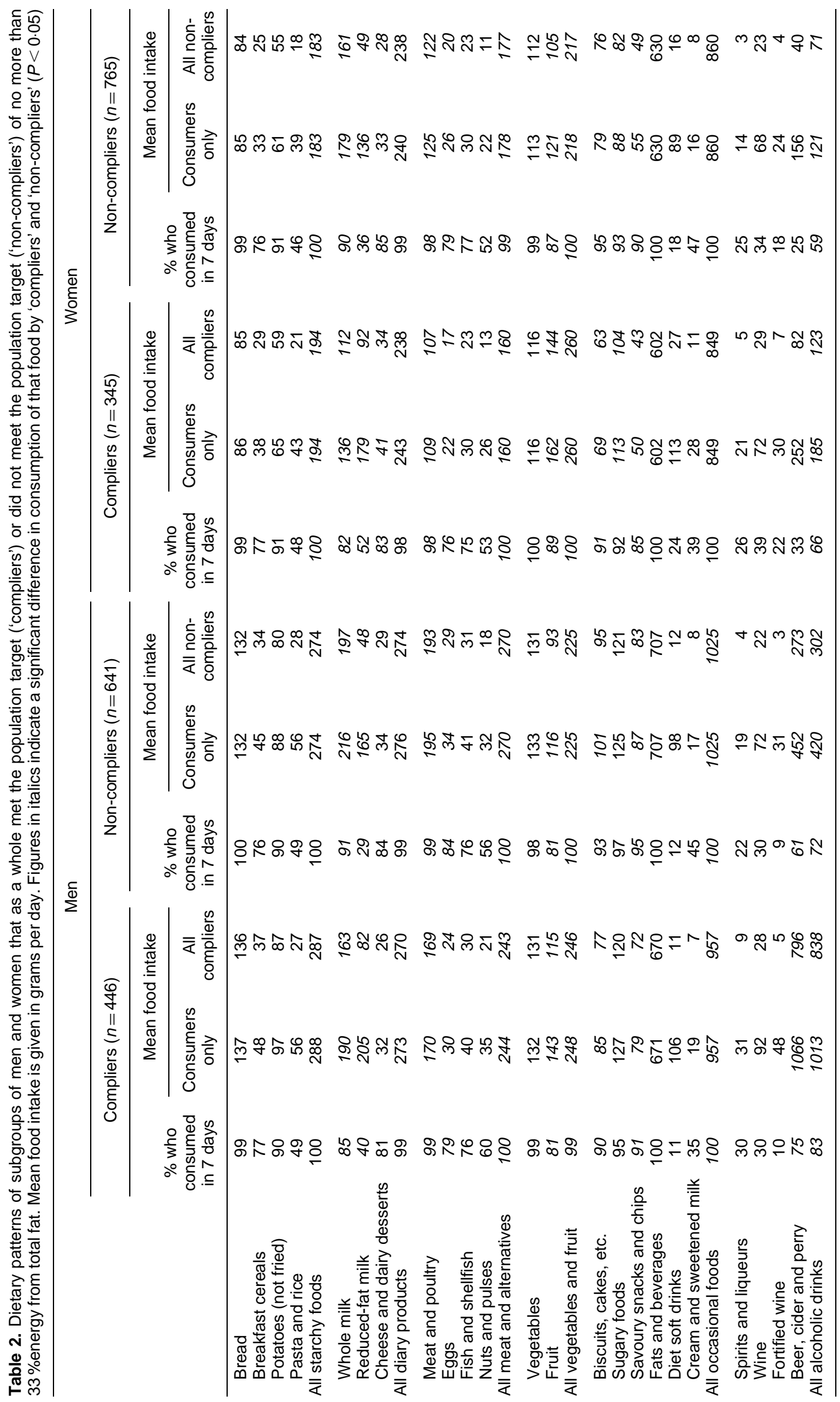




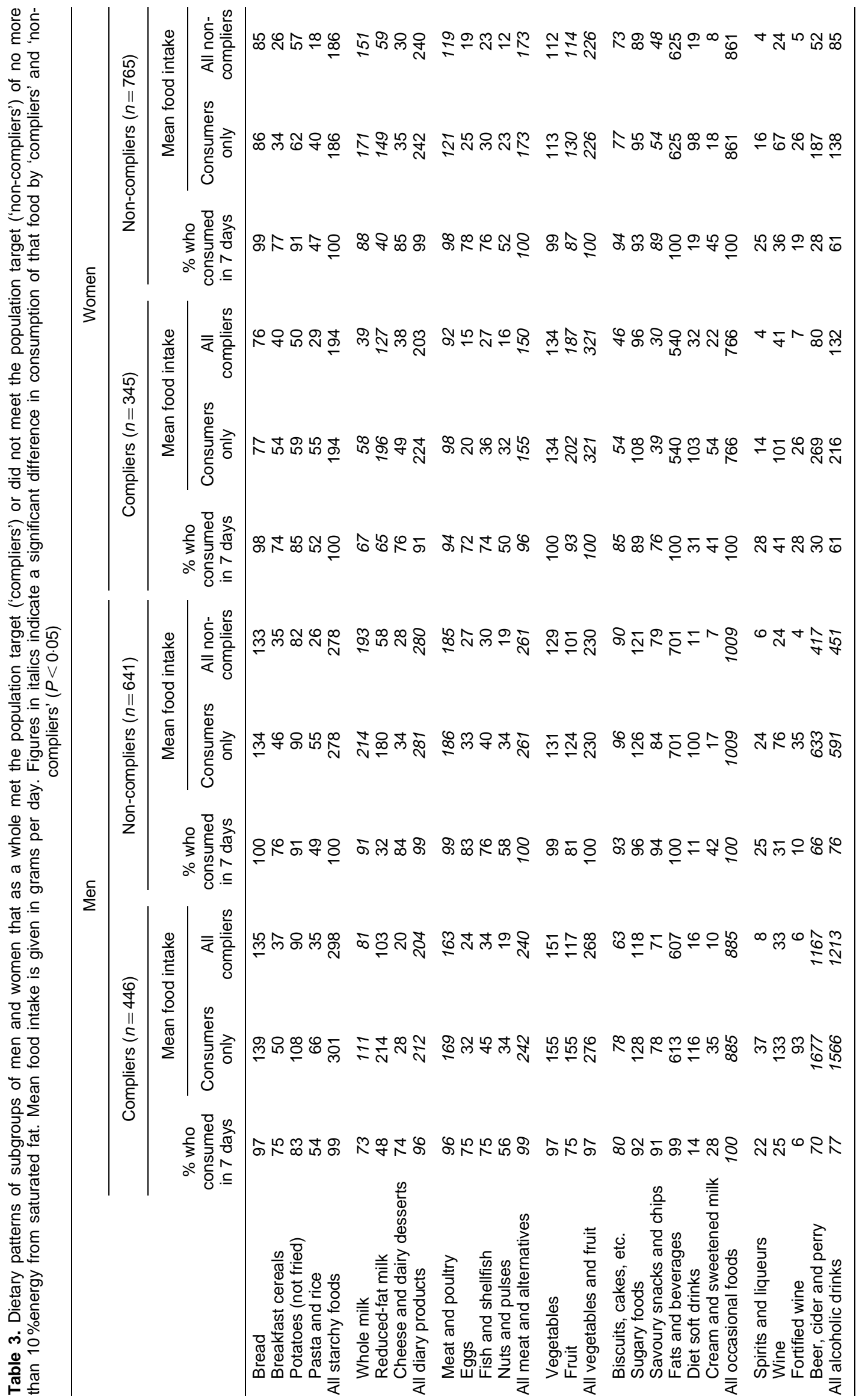




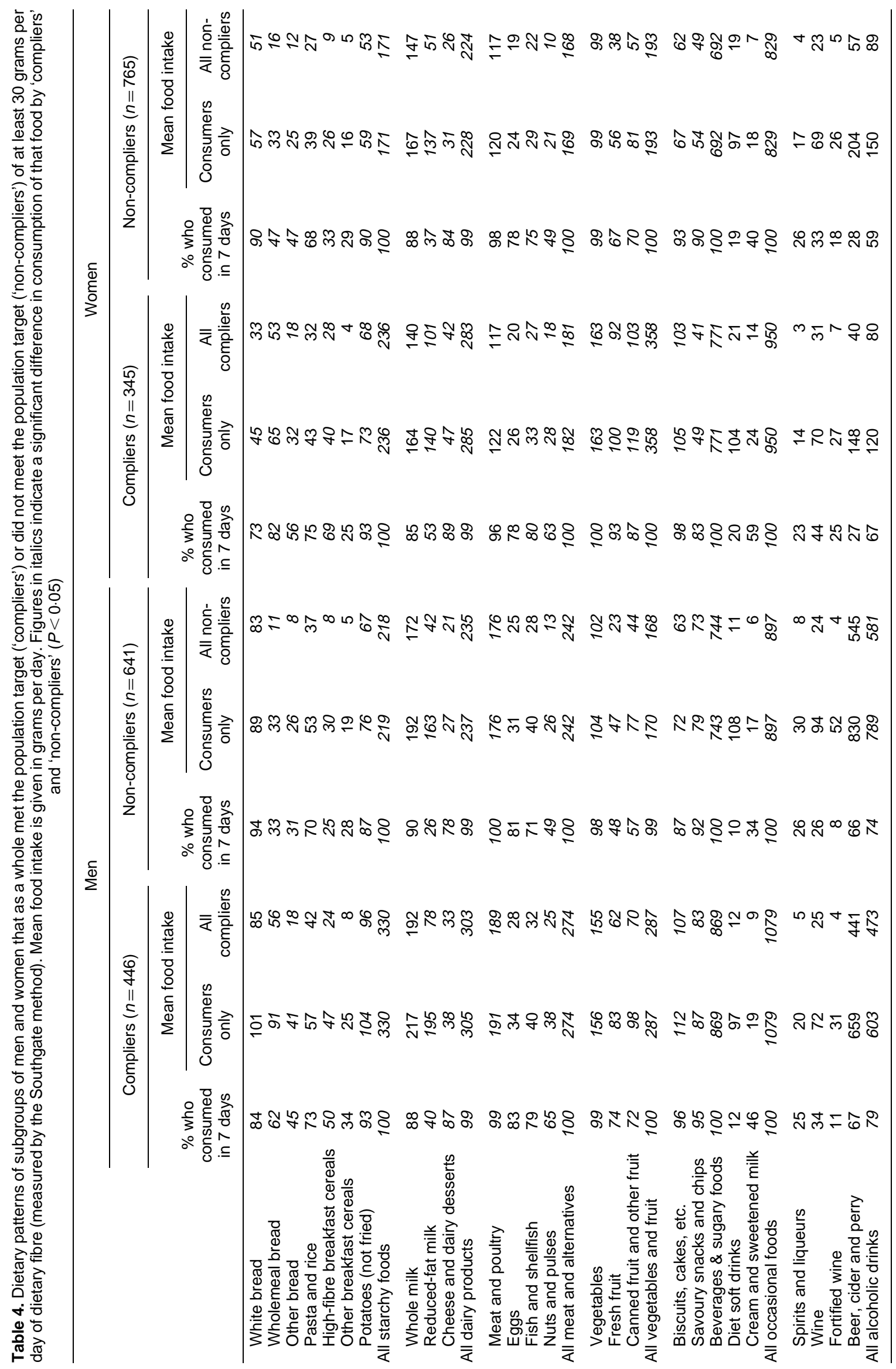


period, mean intakes of vegetables by 'compliers' were significantly higher.

There was also some evidence that people with higher fibre intakes also have healthier dietary habits in general. The proportion of the subgroup that consumed reduced-fat milks and the mean intakes of these products by the people who consumed them were both significantly higher for 'compliers' than for 'non-compliers'.

The analysis may have been in part confounded by the observation that fibre 'compliers' consumed significantly more food and drink overall than fibre 'non-compliers' $(4275 \mathrm{~g} / \mathrm{d}$ vs. $3797 \mathrm{~g} / \mathrm{d}$ for men; $3078 \mathrm{~g} / \mathrm{d}$ vs $2562 \mathrm{~g} / \mathrm{d}$ for women). Indeed, there were significantly higher levels of consumption of beverages and sugary foods amongst 'compliers' when compared to 'non-compliers', which would not have been expected a priori. If these differences in overall food and drink consumption were to be mirrored by differences in total energy intake between 'compliers' and 'non-compliers', this confounding effect might then be reduced by conducting the analysis not in terms of absolute intakes of dietary fibre, but in terms of intakes of dietary fibre per unit of energy. This and other potential confounders, such as differential under-reporting of foods, are discussed in other papers in this volume.

\section{Discussion \\ Dietary habits consistent with the attainment of nutritional goals}

Comparing the above initial analyses of the dietary habits of the different subgroups who meet different nutritional goals allows certain achievable dietary behaviours to be distinguished that are consistent with all three goals - a lower intake of total fat and saturated fat, and a higher intake of dietary fibre. These suggest themselves as natural targets for any refinement of food-based dietary guidelines relevant to the UK population, and may be summarized as: eat more starchy foods (particularly wholemeal varieties), more fruit, and substitute reduced-fat milk for full-fat milk. This is consistent with existing evidence that such a pattern of dietary behaviour is consistent with improved patterns of nutrient intake.

The above analysis also indicates that, for men, a relatively higher consumption of alcoholic beverages is consistent with attainment of the nutritional benchmarks. In terms of dietary fibre goals, analysed here in terms of absolute intakes, this is consistent with the observation that fibre 'compliers' consumed significantly more food and drink overall than fibre 'non-compliers', discussed above. For total and saturated fat targets, which have been analysed in terms of the percentage contribution these nutrients make to total energy intake, the result is unsurprising as alcohol is energy dense and the consumption of alcohol with, say, a meal is likely to significantly increase the overall energy intake from that meal thus significantly decreasing the proportion of the energy intake from nutrients other than alcohol. In terms of alcohol and health, the effects of unwise alcohol consumption have been well documented and, increasingly, the potentially beneficial effects of moderate alcohol consumption for some sectors of the population are being described. These, together with an acknowledgement of the particular social and cultural associations of alcohol consumption, have led to specific public health advice on alcohol consumption being considered in a wider context, taking into account the extensive and disparate evidence on alcohol and health (Department of Health, 1995).

\section{The role of food-based dietary guidelines in campaigns to promote dietary change}

It is increasingly asked whether population-based campaigns promoting dietary change have actual effects on dietary habits. Although there is a wealth of information from intensive individual or small-group interventions in a clinical setting, these do not provide information that is applicable to large-scale community or population-based interventions.

Recent cross-sectional studies have indicated that increased activity in promulgating population-based health promotion programmes has coincided in time with largescale changes in population behaviours (Turrell, 1997). Evidence from the UK is consistent with these findings. Data from the annual National Food Survey (MAFF, 1997) allows year-on-year changes in average population intakes to be assessed. These show that the average percentages of food energy derived from total fat and saturated fat have declined steadily in the decade since 1986, when fieldwork for the Dietary and Nutritional Survey of British Adults described in this paper was conducted, from $42.6 \%$ to $39.7 \%$ for total fat and $17.7 \%$ to $15.4 \%$ for saturated fat. We would therefore expect considerably larger proportions of the population than the proportions given in Table 1 now to be meeting nutritional goals. In addition to these temporal associations, there is also a large degree of consistency in cross-sectional studies that associate healthy dietary behaviours with a knowledge of dietary guidelines.

Together, these studies are insufficient to establish whether health promotion programmes are causally linked to positive changes in dietary behaviour. Evidence from a recent largescale longitudinal study (Patterson et al. 1996) suggests that different types of information may lead to positive changes in dietary behaviour at different stages in individuals' receptivity to the information. Interventions presenting information on the connection between diet and chronic disease may aid the initiation of dietary change in adults contemplating such a change, whereas information on food composition (such as is provided in food-based dietary guidelines like The Balance of Good Health) may help adults already making dietary changes to achieve their nutritional goals. Although considerable caution would need to be exercised in extrapolating these findings to different national and cultural groups, they provide some reassurance that foodbased dietary guidelines have a role to play in effective population-based health promotion.

\section{Acknowledgements}

The authors would like to acknowledge the help provided by Gillian Smithers (MAFF) in checking the definitions of food groups used in this paper, and also other colleagues in MAFF and the Department of Health for their critical evaluation of the manuscript. 


\section{References}

Department of Health (1991) Report on Health and Social Subjects 41: Dietary Reference Values for Food Energy and Nutrients for the United Kingdom. Report of the Panel on Dietary Reference Values of the Committee on Medical Aspects of Food Policy. London: HM Stationery Office.

Department of Health (1992) The Health of the Nation: A Strategy for Health in England. Cm1986. London: HM Stationery Office.

Department of Health (1995) Sensible Drinking: The Report of an Inter-Departmental Working Group. London: Department of Health.

Department of Health (1998) Our Healthier Nation: A Contract for Health. Cm3852. London: The Stationery Office.

Gregory J, Foster K, Tyler H \& Wiseman H (1990) The Dietary and Nutritional Survey of British Adults. London: HM Stationery Office.

Health Education Authority (1997) Eight Guidelines for a Healthy Diet: A Guide for Nutrition Educators. Abingdon: Health Education Authority (in association with the Ministry of Agriculture, Fisheries and Food and the Department of Health).

Hunt P, Gatenby S \& Rayner M (1995) The format for the National Food Guide: performance and preference studies. Journal of Human Nutrition and Dietetics 8, 335-352.
MAFF (Ministry of Agriculture, Fisheries and Food) (1994) The Dietary and Nutritional Survey of British Adults: Further Analysis. London: HM Stationery Office.

MAFF (Ministry of Agriculture, Fisheries and Food) (1997) National Food Survey 1996. London: The Stationery Office.

Patterson RE, Kristal AR \& White E (1996) Do beliefs, knowledge and perceived norms about diet and cancer predict dietary change? American Journal of Public Health 86, 1394-1400.

Research and Auditing Services Ltd (1997) Balance of Good Health comparative research: final report. Unpublished report prepared for the Health Education Authority.

Southgate DAT (1969) Determination of carbohydrates in foods. II. Unavailable carbohydrates. Journal of the Science of Food and Agriculture 20, 331-335.

Turrell G (1997) Compliance with the Australian dietary guidelines in the early 1990s: have population-based health promotion programs been effective? Nutrition and Health 11, 271-288.

World Health Organization (1990) Diet, nutrition and the prevention of chronic diseases: report of a WHO Study Group. Technical Report Series no. 797. Geneva: WHO.

World Health Organization (1998) Preparation and use of foodbased dietary guidelines: report of a joint FAO/WHO consultation. Technical Report Series no. 880. Geneva: WHO. 